\title{
SUR LE DÉNOMBREMENT ET L'IDENTIFICATION DES STAPHYLOCOQUES PATHOGENES DANS LES PRODUITS LAITIERS
}

\author{
par \\ François-M. LUQUET \\ Laboratoire d'Industrie Laitière de Douai
}

\section{I. - Généralités}

Les staphylocoques appartiennent à la famille des micrococcaccae. Ce sont des cocci immobiles de 0,5 à $1 \mu$ de diamètre, grampositifs, qui se présentent généralement en amas. Ils peuvent se multiplier entre $10^{\circ}$ et $45^{\circ} \mathrm{C}$, avec un optimum à $37^{\circ} \mathrm{C}$ pour des valeurs de $p \mathrm{H}$ comprises entre 5 et 9 , avec un optimum à 7,4 . Ils se distinguent des microcoques par leur type respiratoire (aérobieanaérobie facultatif) et par leur action sur le mannitol qu'ils font fermenter ou non suivant les variétés, alors qu'il est oxydé par les microcoques.

Sur milieu Plate Count Agar, les colonies sont régulières, rondes, opaques, de couleur variant du blanc au jaune rougeâtre (Bergey [1]).

Les staphylocoques pathogènes donnent des réactions positives ou négatives suivant les espèces au test de la coagulase, positives aux tests de la catalase, de la phosphatase et de la désoxyribonucléase.

Différentes techniques ont été préconisées pour leur dénombrement et leur identification. En expérimentant ces techniques, et en particulier celles qui sont indiquées dans les réglementations officielles en France [2], nous avons été amenés à les préciser. Le tableau I donne le résumé de la méthode que nous utilisons couramment, et dont les modalités sont décrites ci-après.

\section{II. - Préparation de l'échantillon à analyser}

Homogénéisation et dilution dans la tryptone-sel [3].

Quel que soit le produit à analyser, l'échantillon après homogénéisation est dissous ou mis en suspension dans un bouillon tryptone-sel, préparé de la façon suivante : 
Dissoudre dans $1000 \mathrm{ml}$ d'eau distillée :

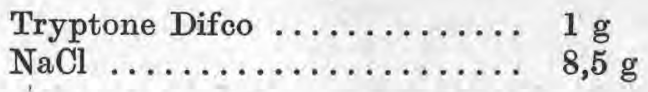

Stériliser à l'autoclave à $120^{\circ} \mathrm{C}$ pendant $20 \mathrm{mn}$.

Ce diluant a le double avantage :

a) De redonner de la vigueur aux germes microbiens qui auraient pu être affaiblis au cours des traitements subis par le produit;

b) D'éviter la multiplication de ces germes si la dilution est utilisée dans l'heure qui suit sa préparation.

La dilution s'effectue en mélangeant de façon aussi homogène que possible [30] :

- Pour les laits en poudre et laits concentrés : $10 \mathrm{~g}$ de produit et $40 \mathrm{ml}$ de diluant;

- Pour les fromages : $10 \mathrm{~g}$ de produit finement broyé dans un mixer et $40 \mathrm{ml}$ de diluant;

- Pour les préparations alimentaires à base de matières grasses (beurres sucrés, par ex.) : $10 \mathrm{~g}$ de produit et $30 \mathrm{ml}$ de diluant;

- Pour les beurres, employer la technique suivante : faire fondre $100 \mathrm{~g}$ de beurre au bain-marie à $45^{\circ} \mathrm{C}$ en agitant régulièrement. Centrifuger pendant $1 \mathrm{mn}$ à $1200 \mathrm{t} / \mathrm{m}$. Porter en chambre froide pendant 15 à $20 \mathrm{mn}$ de façon à provoquer la solidification de la matière grasse. Prélever $10 \mathrm{ml}$ de la phase aqueuse et mélanger à $40 \mathrm{ml}$ de diluant.

\section{III. - Dénombrement des staphylocoques pathogènes}

Le résultat du dénombrement des staphylocoques pathogènes peut s'exprimer de deux façons différentes :

a) Soit par le nombre de germes présents dans $1 \mathrm{~g}$ de produit à analyser, et dans ce cas, on utilisera une méthode de dénombrement direct;

b) Soit par la constatation de l'absence ou de la présence des germes dans une quantité déterminée de produit à analyser, et dans ce cas, on cherchera à provoquer le développement des germes sur un milieu sélectif avant de pratiquer une subculture.

\section{a) Dénombrement direct.}

Le dénombrement direct est effectué en boîtes de Petri, en utilisant le milieu gélosé de Baird Parker (milieu de Zeibovitz, modifié par addition de pyruvate de sodium et de jaune d'œuf). 


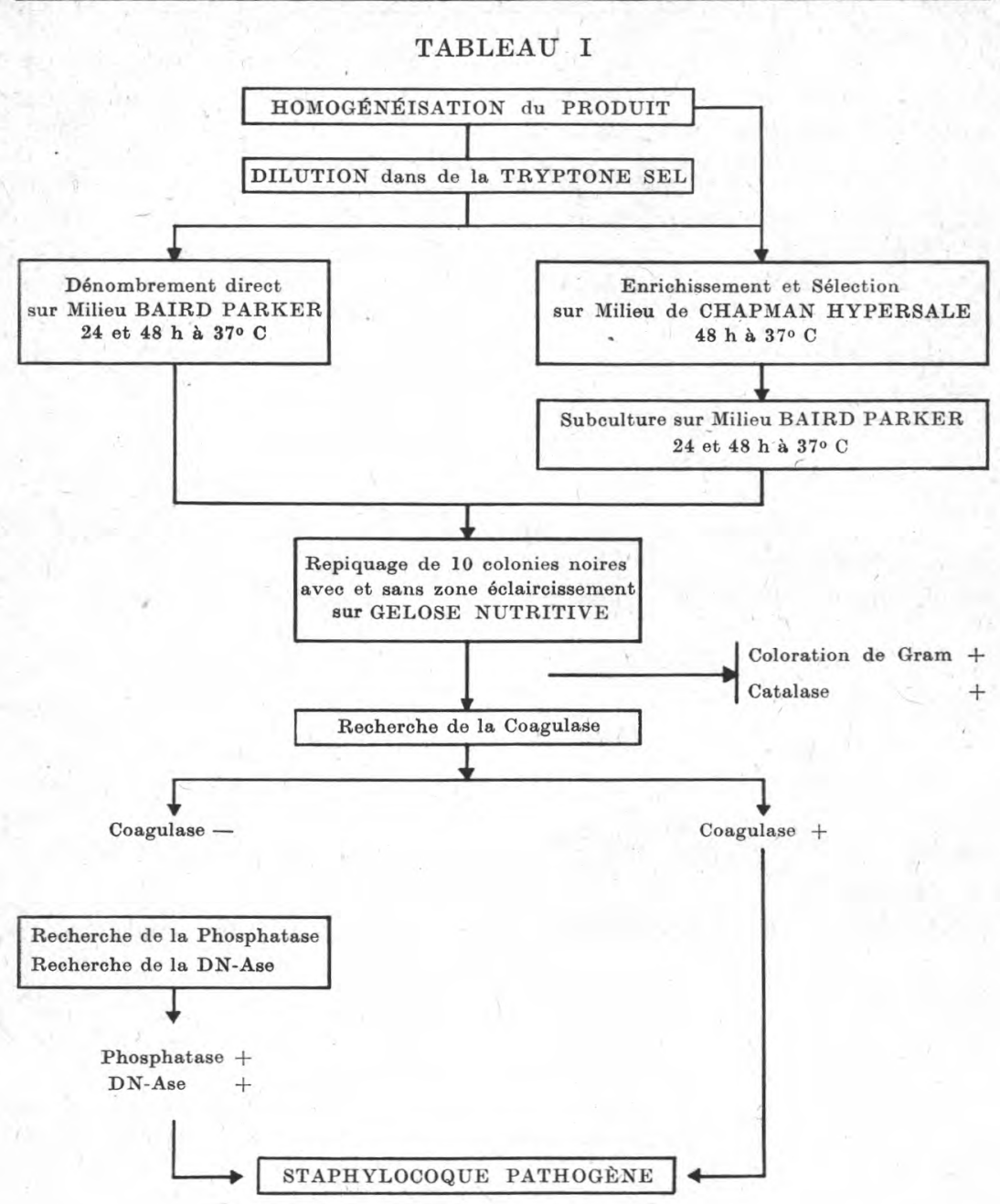

10 Préparation du milieu de Baird Parker:

\begin{tabular}{|c|c|}
\hline ryptone Difco . & $10 \mathrm{~g}$ \\
\hline de viande de bœuf & \\
\hline Extrait de levure $\ldots \ldots \ldots \ldots \ldots \ldots$ & \\
\hline Chlorure de lithium..$\ldots \ldots \ldots \ldots$. & \\
\hline Agar $\ldots \ldots \ldots \ldots$ & $20 \mathrm{~g}$ \\
\hline
\end{tabular}

Ajuster le milieu à $p \mathrm{H} 7,2$. Stériliser à l'autoclave à $120^{\circ} \mathrm{C}$ pendant 20 minutes. Répartir aseptiquement dans des flacons de $200 \mathrm{ml}$, ̀̀ raison de $90 \mathrm{ml}$ par flacon. 
Au moment de l'emploi, faire fondre le milieu et ajouter, lorsqu'il est à une température de 45 à $50^{\circ} \mathrm{C}$, les solutions suivantes portées à la même température :

a) Emulsion concentrée de jaune d'œuf (1) ......... $5 \mathrm{ml}$

b) Solution aqueuse à 1 p. 100 de tellurite de potassium ... I ml

c) Solution aqueuse à 20 p. 100 de pyruvate de sodium $\ldots 5 \mathrm{ml}$

d) Solution aqueuse à $20 \mathrm{p} .100$ de glycine $\ldots \ldots \ldots \ldots \ldots 6 \mathrm{ml}$

Les solutions aqueuses sont stérilisées préalablement par filtration sur membranes millipore stériles (micro Syringe filter Holder XX 30 02500). La filtration se fait en deux temps : préfiltration sur membrane AA $0,8 \mu$ et filtration sur membrane HA $0,4 \mu(2)$.

Au cas où le produit à analyser serait riche en proteus, ajouter $50 \mu \mathrm{g}$ de sulfadimidine ou de sulfamézathine par $\mathrm{ml}$ de milieu (technique préconisée en 1964 par Smith et Baird Parker [4]).

\section{$2^{\circ}$ Utilisation du milieu de Baird Parker.}

Une fois terminé, le milieu doit être utilisé dans les 24 h qui suivent sa préparation (3).

Il est coulé en boîtes de Petri et lorsqu'il est solidifié, on répartit en surface, au moyen d'un étaleur de verre stérile ou d'une ose bouclée, $0,1 \mathrm{ml}$ de la dilution du produit à analyser dans le bouillon tryptone-sel.

Les boîtes de Petri sont portées à l'étuve à une température de $37^{\circ} \mathrm{C} \pm 0,1$. Les dénombrements sont effectués après 24 et $48 \mathrm{~h}$ d'incubation.

\section{Lecture des résultats.}

Après l'incubation, l'on compte les colonies noires rondes d'un diamètre de 1 à 1,5 mm, entourées d'une zone d'éclaircissement.

(1) L'émulsion stérile de jaune d'œuf peut être fournie par Oxoid (Réf. SR 44), ou préparée au laboratoire de la façon suivante :

a) Nettoyer à l'alcool éthylique la coquille d'un œuf de poule très frais ;

b) Crever la coquille au niveau de la chambre à air ;

c) Aspirer le blanc au moyen d'une pipette stérile sans effilure;

d) Aspirer le jaune au moyen d'une pipette stérile et l'émulsionner dans $250 \mathrm{ml}$ de solution de Ringer diluée au 1/4 ou dans $250 \mathrm{ml}$ de solution aqueuse à 0,85 p. 100 de chlorure de sodium;

e) Vérifier la stérilité avant utilisation.

(2) Les solutions peuvent être préparées extemporanément; elles peuvent être conservées au maximum pendant un mois, et à condition d'être maintenues à une température de $4^{\circ} \mathrm{C}$.

(3) On trouve dans le commerce le milieu de Baird Parker sous forme déshydratée (Oxoid CM 275, dépositaire en France Biolyon), mais il faut ajouter au moment de l'emploi l'émulsion de jaune d'œuf et la solution de tellurite de potassium. 
Selon la dilution utilisée, l'on ramène le résultat au $\mathrm{ml}$ de diluant, puis par calcul au g de produit analysé.

Par exemple: Analyse d'un beurre sucré.

10 colonies trouvées sur la boîte (dilution 1/100) correspondent à 1000 colonies au $\mathrm{ml}$ soit $4000 / \mathrm{g}$.

\section{b) Recherche des staphylocoques pathogènes, sans dénom- brement.}

$1^{0}$ Enrichissement et sélection sur milieu de Chapman hypersalé.

Le nombre des germes microbiens revivifiables présents dans un produit laitier est très variable suivant les produits, mais, d'une façon très générale, on peut dire que cette flore revivifiable est surtout composée de ferments lactiques et que les staphylocoques pathogènes, lorsqu'ils sont présents ne constituent la plupart du temps, qu'une très faible partie de la dite flore.

Il y a donc souvent intérêt à inhiber le plus possible le développement des ferments lactiques pour permettre aux staphylocoques de se développer ; on emploie pour ce faire le milieu sélectif hypersalé de Chapman que l'on prépare de la façon suivante (1) : rement :

a) Dissoudre dans $500 \mathrm{ml}$ d'eau distillée, en chauffant légè-

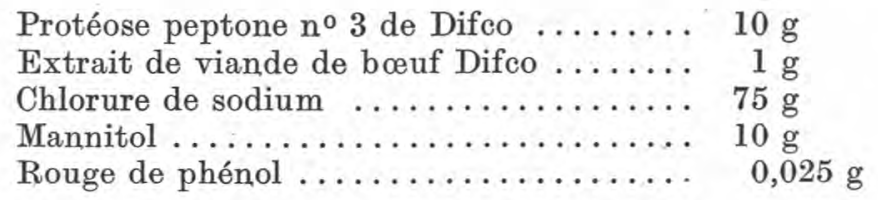

b) Ajuster à $1000 \mathrm{ml}$ au moyen d'eau distillée ;

c) Ajuster le milieu à $p \mathrm{H} 7,4$;

d) Stériliser à l'autoclave à $120^{\circ} \mathrm{C}$ pendant $20 \mathrm{mn}$;

e) Répartir aseptiquement en tubes stériles de $160 \times 16$ à raison de $9 \mathrm{ml}$; ou en ballons stériles de $150 \mathrm{ml}$ à raison de $90 \mathrm{ml}$ (2).

Le produit à examiner, qui peut être ou non dilué dans la tryptone-sel, est ensemencé suivant les cas :

- Soit à raison de 1 ou $2 \mathrm{~g}$ dans les tubes;

- Soit à raison de $5 \mathrm{~g}$ dans les ballon,s.

(1) On trouve dans le commerce le milieu de Chapman déshydraté, soit chez Mérieux (Réf. BTB 01712), soit chez Difeo (Chapman Stone medium 01316).

(2) Voir note à la page suivante. 
On porte à l'étuve à $30^{\circ} \mathrm{C}$ pendant $24 \mathrm{~h}$, puis à l'étuve à $37^{\circ} \mathrm{C}$ pendant $24 \mathrm{~h}$.

$2^{\circ}$ Subcultures sur milieu solide de Baird Parker.

La culture d'enrichissement est ensemencée sur milieu de Baird Parker suivant les modalités indiquées au paragraphe $a$ ) $2^{\circ}$ ci-dessus.

$3^{\circ}$ Aspect des colonies sur le milieu de Baird Parker.

a) Les microcoques se multiplient rarement sur le milieu de Baird Parker. Cependant ils peuvent donner parfois de petites colonies noires ou brunes sans zone d'éclaircissement.

b) Les levures donnent des colonies blanches, sans zone d'éclaircissement.

c) Quelques variétés de bacillus donnent des colonies mates, brunes, la zone d'éclaircissement n'apparaissant qu'après $48 \mathrm{~h}$ d'incubation.

d) Les staphylocoques pathogènes se présentent sous forme de colonies noires, rondes, convexes, d'un diamètre de 1 à $1,5 \mathrm{~mm}$. Elles sont entourées d'une zone ronde d'éclaircissement de 2 à $5 \mathrm{~mm}$ de diamètre.

Certains auteurs $\left[\begin{array}{lll}6 & \text { et } 15 & b i s\end{array}\right]$ ont noté que certaines colonies de staphylocoques pathogènes ne présentaient pas de zone d'éclaircissement ou que l'éclaircissement n'était net qu'après 36 ou 48 h d'incubation.

Note de la page 47.

(2) Si l'on veut vérifier l'absence des staphylocoques pathogènes dans une quantité importante de produits ( 10 ou $20 \mathrm{~g}$ par exemple), exigence qui figure dans certaines réglementations étrangères, il est préférable d'utiliser le milieu de Chapman modifié par Buttiaux et Brogniart [5]; ce milieu est préparé de la façon suivante :

a) Dissoudre $500 \mathrm{ml}$ d'eau distillée en chauffant légèrement :

\begin{tabular}{|c|c|}
\hline Protéose peptone no 3 Difco ......... & $15 \mathrm{~g}$ \\
\hline Extrait de viande de bœuf Difco & \\
\hline Chlorure de sodium ........... & 150 \\
\hline Lactose $\ldots \ldots \ldots \ldots \ldots \ldots \ldots \ldots$ & 15 \\
\hline Agar $\ldots \ldots \ldots \ldots \ldots \ldots \ldots \ldots$ & 2 \\
\hline
\end{tabular}

b) Ajuster à $1000 \mathrm{ml}$ au moyen d'eau distillée;

c) Ajuster le milieu à $p H \quad 7,4$;

d) Répartir en ballons de $200 \mathrm{ml}$ à raison de $50 \mathrm{ml}$;

e) Stériliser à l'autoclave à $120^{\circ} \mathrm{C}$ pendant 20 minutes.

L'échantillon à examiner est dilué dans le bouillon tryptone sel (10 ou $20 \mathrm{~g}$ de produit + tryptone sel $=50 \mathrm{ml}$ ).

Les $50 \mathrm{ml}$ ainsi obtenus sont versés dans un ballon de $200 \mathrm{ml}$ contenant $50 \mathrm{ml}$ de milieu. On porte à l'étuve à $30^{\circ} \mathrm{C}$ pendant 24 heures, puis à l'étuve à $37^{\circ} \mathrm{C}$ pendant 24 heures. 


\section{IV. - Identification des staphylocoques pathogènes}

\section{a) Repiquage.}

On repique 10 colonies ayant l'aspect indiqué ci-dessus, sur des tubes inclinés de gélose nutritive qui peut être :

- Soit le milieu Difco B 37 ;

- Soit un milieu préparé au laboratoire comme suit : dissoudre dans $1000 \mathrm{ml}$ d'eau distillée :

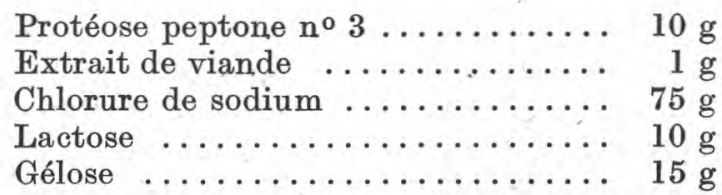

Ajuster à $p H 7,4$, répartir à raison de $5 \mathrm{ml}$ par tubes de $160 \times 16$, stériliser à l'autoclave à $120^{\circ} \mathrm{C}$ pendant $15 \mathrm{mn}$, laisser refroidir en inclinant les tubes.

Après ensemencement, les tubes sont portés à l'étuve pendant $24 \mathrm{~h}$ à $37^{\circ} \mathrm{C}$.

\section{b) Examen des colonies.}

10 Examen au microscope.

Les colonies sont examinées au microscope après coloration au gram. Les staphylocoques pathogènes sont gram + .

2 - Test de la catalase.

Si la morphologie n'est pas caractéristique, il est recommandé d'effectuer le test de la catalase. Les staphylocoques pathogènes donnent une réaction positive.

$3^{\circ}$ Recherche de la coagulase [16 à 29].

La coagulase libérée par les staphylocoques pathogènes est mise en évidence de la façon suivante :

Dans un tube stérile de $120 \times 8$, introduire aseptiquement, 5 gouttes de plasma humain ou de plasma de lapin, que l'on se procure sous forme déshydratée :

- soit à l'Institut Pasteur (reconstitution 1 flacon 286 dans $3 \mathrm{ml}$ d'eau) ;

- Soit chez Difco (Réf. 286-33);

- Soit chez Biolyon ou Biotrol. 
Ajouter 5 gouttes d'une suspension du germe à étudier. Agiter. Incuber 2 à $24 \mathrm{~h}$ à $37^{\circ} \mathrm{C}$. La présence de coagulase a pour effet de solidifier le plasma. En général, cette coagulation apparaît pour les staphylocoques pathogènes au bout de 2 à $3 \mathrm{~h}$.

La présence de coagulase permet d'affirmer que la colonie de staphylocoque étudiée est bien pathogène.

Si la recherche de la coagulase est négative, il faut procéder à celles de la phosphatase et de la désoxyribonucléase.

\section{$4^{\circ}$ Recherche de la phosphatase.}

Différentes techniques ont été proposées, en particulier par Barber et Kuper [7], White et Pickett [8], etc.

Pour notre part, nous utilisons les tablettes "Phosphatabs Acid" de la Société Warner-Chilcott (diffusion en France par AlcaSynthése ou Precibio [9]) suivant les modalités ci-après :

La souche à étudier est dilacérée dans un tube à essai contenant $0,5 \mathrm{ml}$ d'eau salée stérile à 9 p. 100 ; ajouter un comprimé d' $\alpha$ naphthyl phosphate acide de sodium; incuber $30 \mathrm{mn}$ à $37^{\circ} \mathrm{C}$; ajouter un comprimé d'orthodianisidine bis-di-azotée.

La présence de phosphatase se traduit par une coloration rose sur les bords du tube (1).

\section{$5^{\circ}$ Recherche de la désoxyribonucléase.}

La désoxyribonucléase est une diastase sécrétée par un certain nombre d'espèces microbiennes (levures, moisissures) et en particulier par les staphylocoques pathogènes [10], [11], [12]. Pour la mettre en évidence, on utilise un milieu que l'on se procure chez Merieux (B.B.L.) ou que l'on prépare au laboratoire.

a) Préparation du milieu de base:

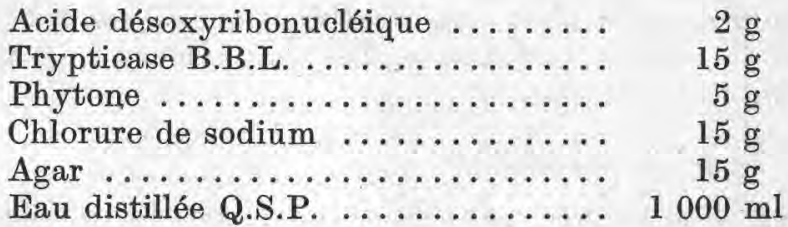

Chauffer en agitant et maintenir à l'ébullition pendant $1 \mathrm{mn}$; ajuster à $p H \quad 7,3$; stériliser à l'autoclave à $120^{\circ} \mathrm{C}$ pendant $15 \mathrm{mn}$.

(1) La Société Precibio vient de mettre sur le marché une technique améliorée en rapidité, précision et stabilité de coloration relative à l'emploi de phosphastrate. Certains auteurs (31) ont mis au point une méthode de dosage rapide (gain de $24 \mathrm{~h}$ ) de la phosphatase. Ils colorent par voie directe sur le milieu de Baird Parker. 


\section{b) Utilisation du milieu:}

Couler en boîtes de Petri ; ensemencer par strie (du centre vers les bords) ; porter à l'étuve à $37^{\circ} \mathrm{C}$ pendant 24 puis $48 \mathrm{~h}$.

c) Mise en évidence de la désoxyribonucléase:

Après incubation, répartir dans les boîtes de Petri $1 \mathrm{ml}$ d'acide chlorhydrique normal ou de solution aqueuse à 1 p. 100 de bleu de toluidine (pour bactériologie).

La désoxyribonucléase se manifeste par l'apparition au bout de $5 \mathrm{mn}$ :

- Soit d'une zone claire autour de la strie, quand on a utilisé l'acide chlorhydrique ;

- Soit d'une zone rose autour de la strie, le reste de la boîte restant bleu quand on a utilisé le bleu de toluidine.

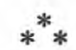

La méthode décrite ci-dessus convient pour des travaux de contrôle. Les laboratoires de recherche qui veulent confirmer scientifiquement leur diagnostic, devront effectuer :

a) Des essais sur animaux;

b) Une galerie d'identification plus complète ;

c) La recherche des toxines staphylococciques : exotoxines (hémolysines $\alpha$ et $\beta$ ) ; entérotoxines A et B [13].

d) Le typage sérologique et le typage par les bactériophages spécifiques [14].

\section{Résumé}

L'auteur décrit une méthode de contrôle employée pour la recherche des staphylocoques pathogènes dans le lait et les produits laitiers pour leur dénombrement et leur identification. Il préconise une culture sur milieu de Baird Parker, dont les résultats doivent être confirmés par le test de la coagulase, et dans le cas où celui-ci est négatif par les tests de la phosphatase et de désoxyribonucléase.

Note de l'auteur. - Profitant de l'anaérobiose facultative des staphylocoques, il serait possible d'utiliser, pour le dénombrement ou la recherche de ce germe, la technique de GreEnBerg $(32,33)$ en utilisant des sachets très minces de Straboplex FE 1550. Cette technique évite l'utilisation de boîtes de Petri et permet d'abaisser très sensiblement le prix de revient de l'analyse.

Des essais sont en cours dans nos laboratoires et seront l'objet d'une prochaine publication. 


\section{Summary}

The author describes a control method used for the detection, the count and identification of pathogenic staphylococoi in milk and milk products. The Baird Parker culture medium is recommended; the results should be controlled by the coagulase test and, if the latter is negative, by the phosphatase and deoxyribonuclease tests.

\section{BIBLIOGRAPHIE}

[1] Bergey's Manual of determinative bacteriology.

[2] "Répression des fraudes", J.O., 1963, 1232.

[3] Mossel, Annales I.P.L., 1965, 16, 119.

[4] Batrd Parker (A. C.). J. Appl. Bact., 1962, 25, 12 et 441.

[5] Buttiaux (R.) et Brogmiard (R.). Annales de l'Institut Pasteur, 1947, 73, 830 .

[6] Ten Brocke (R.), D, Mossel (A. A.), VAN de MoosdiJk (A.). Le milieu de Baird Parker en pratique courante. La fréquence des staphylocoques, coagulase + , jaune d'œuf + .

[7] Barber (M.) et KuPER (S. W. A.). J. Patho. Bact., 1951, 63, 65.

[8] White (M. L.) et Picketw (M. J.). Amer. J. Clin. Path., 1953, 23, 1181.

(9) Babson (A. L.) : Read. P.A. and Phillips G.E. Am. J. Clin. Path., 1959,32 , I (July).

[10] WECKMAN et CATLIN. J. Bact., 1957, 73, 747.

[11] Dr Salvo. Med. Tech. Bull. 1958, 9, 191.

[12] Fussinlo et Werss. J. Bact., 1959, 78, 520.

[13] Casman et Bennetr. Applied Microbiology, 1965, 13, 181.

[14] Williams et Rippon. J. Hyg., 1952, 50, 320.

[15] ButtiauX, Benrens, Tacquet. Manuel de techniques bactériologiques.

[15 bis] ThieULin (G.) et Al. Le Lait, 1966, t. XLVI, 453, 454, 131.

[16] Annales de la Nutrition et de l'Alimentation, 1961, XV, 6.

[16 bis] Lоев. J. Med. Research, 1903, 10, 407.

[17] Brochem. Ztschr., 1908, 14, 143.

[18] J. Bact., 1934, 28, 343.

[19] Proc. Soc. Exp. Biol. Med., 1937, 36, 729.

[20] J. Path. Bact., 1937, 45, 295.

[21] J. Bact., 1938, 35, 311.

[22] J. Path. Bact., 1938, 46, 31.

[23] J. Path. Bact., 1939, 48, 169.

[24] J. Bact. Rev., 1939, 3, 97.

[25] J. Path. Bact., 1940, 50, 83.

[26] J. Bact., 1941, 41, 431.

[27] Am.J. Clin. Path., 1941, 11, 857.

[28] Caduers, Graves, Wilitams, Harpes et Miles. Lancet, 1943, 1, 736.

[29] Nemdham, Ferris and SpInk. Am. J. Clin. Path. Tech. Supp. 9, 1945, $3,83$.

[30] Edward (F. Baer). J. Ass. Of. Anal. Chemist, 1966, IV, 49.

[31] Carassus, Lefranc (C.), Guillaume (J.). Annales I.P.L., 1966, XVII, 57.

[32] Bladel (B. O.) et Greenberg. Applied Microbiology, 1965, 13, 281.

[33] Greenberg (R. A.), Bladel (B. O.) et Zingelmann. Appl. Micro., 1966, XIV, 223. 